\title{
Approximate Solution of Time-Fractional Advection-Dispersion Equation via Fractional Variational Iteration Method
}

\author{
Birol İbiş ${ }^{1}$ and Mustafa Bayram² \\ ${ }^{1}$ Department of Basic Sciences, Turkish Air Force Academy, Istanbul, Turkey \\ ${ }^{2}$ Department of Mathematical Engineering, Faculty of Chemical and Metallurgical Engineering, \\ Yıldız Technical University, Istanbul, Turkey \\ Correspondence should be addressed to Birol İbiş; b.ibis@hho.edu.tr
}

Received 12 September 2013; Accepted 30 October 2013; Published 22 January 2014

Academic Editors: A. Atangana, A. M. A. El-Sayed, A. Kılıçman, S. C. O. Noutchie, S. S. Ray, and A. Secer

Copyright (C) 2014 B. İbiş and M. Bayram. This is an open access article distributed under the Creative Commons Attribution License, which permits unrestricted use, distribution, and reproduction in any medium, provided the original work is properly cited.

This paper aims to obtain the approximate solution of time-fractional advection-dispersion equation (FADE) involving Jumarie's modification of Riemann-Liouville derivative by the fractional variational iteration method (FVIM). FVIM provides an analytical approximate solution in the form of a convergent series. Some examples are given and the results indicate that the FVIM is of high accuracy, more efficient, and more convenient for solving time FADEs.

\section{Introduction}

Many problems in mechanical engineering, physics, biology, chemistry, control theory, fluid mechanics, signal processing, viscoelasticity, electromagnetism, electrochemistry, thermal engineering, and many other physical processes are modeled by fractional differential equations (FDEs) or fractional partial differential equations (FPDEs) [1-9]. The solution of FDEs has been recently studied. In most cases, FDEs do not have analytical solutions, so these equations have been solved by using various analytical and numerical methods. The timefractional advection-dispersion equations, which are a special type of FPDEs, have been applied to many problems [1018]. There may be several methods for solving FADEs such as variable transformation [19], finite element method [20], Adomian decomposition method (ADM) [21], implicit and explicit difference method [22], homotopy analysis method (HAM) [23], optimal homotopy asymptotic method [24], homotopy perturbation method (HPM) [25], Green function [26], and least-squares spectral method [27].

In this study, the following time FADE with the initial condition is discussed:

$$
\begin{array}{r}
\frac{\partial^{\alpha} u(x, t)}{\partial t^{\alpha}}=\kappa(x, t) \frac{\partial^{2} u(x, t)}{\partial x^{2}}+\nu(x, t) \frac{\partial u(x, t)}{\partial x}+g(x, t), \\
x>0, t>0,0<\alpha \leq 1,
\end{array}
$$

$$
u(x, 0)=u_{0}(x),
$$

where $(x, t), \kappa(x, t)$, and $\nu(x, t)$ represent the solute concentration, the dispersion coefficient, and the average fluid velocity, respectively.

\section{Preliminaries}

Some necessary definitions, lemmas, and properties of the fractional calculus are reviewed in this section $[28,29]$.

Definition 1. The Riemann-Liouville fractional integral of order $\alpha$ is defined as

$$
{ }_{0} I_{x}^{\alpha} u(x)=\frac{1}{\Gamma(\alpha)} \int_{0}^{x}(x-\xi)^{\alpha-1} u(\xi) d \xi, \quad x>0,
$$

where $\alpha \geq 0, f \in C_{\mu}, \mu \geq-1$.

Definition 2. The modified Riemann-Liouville fractional derivative of order $\alpha$ is defined as

$$
\begin{array}{r}
{ }_{0} D_{x}^{\alpha} f(x)=\frac{1}{\Gamma(n-\alpha)} \frac{d^{n}}{d x^{n}} \int_{0}^{x}(x-\xi)^{n-\alpha}(f(\xi)-f(0)) d \xi, \\
0 \leq x \leq 1, n \in Z^{+}, n-1 \leq \alpha<n .
\end{array}
$$


The properties of the modified Riemann-Liouville fractional derivative are

(i) product rule for fractional derivatives:

$$
{ }_{0} D_{x}^{\alpha}(u v)=\left({ }_{0} D_{x}^{\alpha} u\right) v+u\left({ }_{0} D_{x}^{\alpha} v\right)
$$

(ii) fractional Leibniz formula:

$$
{ }_{0} I_{x{ }_{0}}^{\alpha} D_{x}^{\alpha} u(x)=u(x)-u(0), \quad 0<\alpha \leq 1,
$$

(iii) integration by parts for fractional order:

$$
{ }_{a} I_{b}^{\alpha}\left[\left(D_{x}^{\alpha} u\right) v\right]=\left.(u v)\right|_{a} ^{b}-{ }_{a} I_{b}^{\alpha}\left[u\left(D_{x}^{\alpha} v\right)\right]
$$

Definition 3. Limit form of the fractional derivative is defined as

$$
u^{(\alpha)}=\lim _{h \rightarrow 0} \frac{\Delta^{\alpha}[u(x)-u(0)]}{h^{\alpha}} .
$$

Definition 4. Fractional derivative is defined for compounded functions as follows:

$$
d^{\alpha} u \cong \Gamma(1+\alpha) d u, \quad 0<\alpha<1 .
$$

Definition 5. The integral with respect to $(d x)^{\alpha}$ is defined as

$$
d u \cong u(x)(d x)^{\alpha}, \quad x \geq 0, u(0)=0,0<\alpha<1 .
$$

Definition 6. The following equality is provided for the continuous function $u: R \rightarrow R$ and $x \rightarrow u(x)$ has a fractional derivative of order $k \alpha\left(k \in Z^{+}\right.$and $\left.0<\alpha \leq 1\right)$ :

$$
u(x+h)=\sum_{k=0}^{\infty} \frac{h^{\alpha k}}{(\alpha k) !} u^{\alpha k}(x)
$$

where $u^{(\alpha k)}$ is the derivative of order $k \alpha$ of $u(x)$.

When substituting $h \rightarrow x$ and $x \rightarrow 0$ in (10), we get the fractional McLaurin series:

$$
u(x)=\sum_{k=0}^{\infty} \frac{x^{\alpha k}}{(\alpha k) !} u^{\alpha k}(0) .
$$

Lemma 7. The solution of continuous function $f(x)$ in (8) is

$$
u=\int_{0}^{x} u(\xi)(d \xi)^{\alpha}=\alpha \int_{0}^{x}(x-\xi)^{\alpha-1} u(\xi) d \xi, \quad 0<\alpha \leq 1
$$

For example, when (12) is applied for function $u(x)=x^{\beta}$, one gets

$$
\begin{array}{r}
\int_{0}^{x} u(\xi)(d \xi)^{\alpha}=\int_{0}^{x} \xi^{\beta}(d \xi)^{\alpha}=\frac{\Gamma(\alpha+1) \Gamma(\beta+1)}{\Gamma(\alpha+\beta+1)} x^{\alpha+\beta} \\
0<x \leq 1 .
\end{array}
$$

\section{Fractional Variation Iteration Method (FVIM)}

Equation (1) with initial conditions is considered to describe the solution procedure of the FVIM. Using the VIM developed by He [30], a correction function for (1) can be set as follows:

$$
\begin{aligned}
u_{n+1}(x, t)= & u_{n}(x, t) \\
+ & I^{\alpha}\left[\lambda ( x , t ) \left(\frac{\partial^{\alpha} u_{n}(x, t)}{\partial t^{\alpha}}-v(x, t) \frac{\partial u_{n}(x, t)}{\partial x}\right.\right. \\
& \left.\left.-\kappa(x, t) \frac{\partial^{2} u_{n}(x, t)}{\partial x^{2}}-g(x, t)\right)\right],
\end{aligned}
$$

$$
\begin{gathered}
u_{n+1}(x, t)=u_{n}(x, t) \\
+\frac{1}{\Gamma(\alpha)} \int_{0}^{t}(t-\xi)^{\alpha-1} \lambda(x, \xi) \\
\times\left(\frac{\partial^{\alpha} u_{n}(x, \xi)}{\partial \xi^{\alpha}}-\nu(x, \xi) \frac{\partial u_{n}(x, \xi)}{\partial x}\right. \\
-\kappa(x, \xi) \frac{\partial^{2} u_{n}(x, \xi)}{\partial x^{2}} \\
-g(x, \xi)) d \xi .
\end{gathered}
$$

New correction functional is obtained as follows by combining (12) and (15):

$$
\begin{aligned}
u_{n+1}(x, t)= & u_{n}(x, t) \\
+\frac{1}{\Gamma(\alpha+1)} \int_{0}^{t} \lambda(x, \xi) & \\
& \times\left(\frac{\partial^{\alpha} u_{n}(x, \xi)}{\partial \xi^{\alpha}}-v(x, \xi) \frac{\partial u_{n}(x, \xi)}{\partial x}\right. \\
& -\kappa(x, \xi) \frac{\partial^{2} u_{n}(x, \xi)}{\partial x^{2}} \\
& -g(x, \xi))(d \xi)^{\alpha}
\end{aligned}
$$

where $\lambda$ is a Lagrange multiplier which can be determined optimally through the variational theory. Here $\left(\widetilde{u}_{n}\right)_{x}$ and $\left(\widetilde{u}_{n}\right)_{x x}$ are considered as restricted variations; that is, $\delta \widetilde{u}_{n}=0$. Making the above functional stationary,

$\delta u_{n+1}(x, t)=\delta u_{n}(x, t)$

$$
+\frac{\delta}{\Gamma(\alpha+1)} \int_{o}^{t} \lambda(x, \xi)
$$




$$
\begin{gathered}
\times\left(\frac{\partial^{\alpha} u_{n}(x, \xi)}{\partial \xi^{\alpha}}-v(x, \xi) \frac{\partial \widetilde{u}_{n}(x, \xi)}{\partial x}\right. \\
-\kappa(x, \xi) \frac{\partial^{2} \tilde{u}_{n}(x, \xi)}{\partial x^{2}} \\
-g(x, \xi))(d \xi)^{\alpha}, \\
\delta u_{n+1}(x, t)=\delta u_{n}(x, t)+\left.\lambda \delta u_{n}(x, \xi)\right|_{\xi=t} \\
-\frac{1}{\Gamma(\alpha+1)} \int_{0}^{t} \frac{\partial^{\alpha} \lambda(x, \xi)}{\partial \xi^{\alpha}} \delta u_{n}(x, \xi)(d \xi)^{\alpha},
\end{gathered}
$$

with the property from (4) and (6), $\lambda(x, \xi)$ must satisfy

$$
\frac{\partial^{\alpha} \lambda(x, \xi)}{\partial \xi^{\alpha}}=0, \quad 1+\left.\lambda(x, \xi)\right|_{\xi=t}=0 .
$$

Therefore, $\lambda(x, \xi)$ is determined as

$$
\lambda(x, \xi)=-1
$$

Substituting (19) into the functional (16) gives the iteration formulation as

$$
\begin{gathered}
u_{n+1}(x, t)=u_{n}(x, t) \\
-\frac{1}{\Gamma(\alpha+1)} \int_{0}^{t}\left(\frac{\partial^{\alpha} u_{n}(x, \xi)}{\partial \xi^{\alpha}}-v(x, \xi) \frac{\partial u_{n}(x, \xi)}{\partial x}\right. \\
-\kappa(x, \xi) \frac{\partial^{2} u_{n}(x, \xi)}{\partial x^{2}} \\
-g(x, \xi))(d \xi)^{\alpha} .
\end{gathered}
$$

We start by selecting an appropriate initial function $u_{0}(x, t)$; the consecutive approximations $u_{n}(x, t)$ of $u(x, t)$ can be easily achieved. Generally, the initial values are chosen as zeroth approximation $u_{0}(x, t)$. Consequently, the solution $u(x, t)$ of $(1)$ is obtained by $(x, t)=\lim _{n \rightarrow \infty} u_{n}(x, t)$.

\section{Approximate Solutions of Time FADEs}

In this section, in order to show the applicability and efficiency of the FVIM for solving time FADEs, some illustrative examples are given.

Example 8. Firstly, the following time FADE subject to the initial condition is considered [24]:

$$
\begin{gathered}
\frac{\partial^{\alpha} u(x, t)}{\partial t^{\alpha}}=\mu \frac{\partial^{2} u(x, t)}{\partial x^{2}}-\frac{\partial u(x, t)}{\partial x}, \quad t>0,0<\alpha \leq 1, \\
u(x, 0)=e^{-x} .
\end{gathered}
$$

The corresponding iterative formula (20) for (21) can be derived as

$$
\begin{array}{r}
u_{n+1}(x, t)=u_{n}(x, t) \\
-\frac{1}{\Gamma(\alpha+1)} \int_{0}^{t}\left(\frac{\partial^{\alpha} u_{n}(x, \xi)}{\partial \xi^{\alpha}}+\frac{\partial u_{n}(x, \xi)}{\partial x}\right. \\
\left.-\mu \frac{\partial^{2} u_{n}(x, \xi)}{\partial x^{2}}\right)(d \xi)^{\alpha} .
\end{array}
$$

Starting with $u_{0}(x, t)=u(x, 0)=e^{-x}$, by the iterative formula (22), we derive the following results:

$$
\begin{array}{r}
u_{1}(x, t)=e^{-x}-\frac{1}{\Gamma(\alpha+1)} \int_{0}^{t}\left(\frac{\partial^{\alpha} u_{0}(x, \xi)}{\partial \xi^{\alpha}}+\frac{\partial u_{0}(x, \xi)}{\partial x}\right. \\
\left.-\mu \frac{\partial^{2} u_{0}(x, \xi)}{\partial x^{2}}\right)(d \xi)^{\alpha}
\end{array}
$$

$$
\begin{aligned}
&= e^{-x}+e^{-x} \frac{(1+\mu)}{\Gamma(\alpha+1)} \int_{0}^{t}(d \xi)^{\alpha} \\
&= e^{-x}\left[1+\frac{(1+\mu)}{\Gamma(\alpha+1)} t^{\alpha}\right], \\
& u_{2}(x, t)=e^{-x}\left[1+\frac{(1+\mu)}{\Gamma(\alpha+1)} t^{\alpha}+\frac{(1+\mu)^{2}}{\Gamma(2 \alpha+1)} t^{2 \alpha}\right], \\
& u_{3}(x, t)=e^{-x}\left[1+\frac{(1+\mu)}{\Gamma(\alpha+1)} t^{\alpha}+\frac{(1+\mu)^{2}}{\Gamma(2 \alpha+1)} t^{2 \alpha}\right. \\
&\left.+\frac{(1+\mu)^{3}}{\Gamma(3 \alpha+1)} t^{3 \alpha}\right]
\end{aligned}
$$

Consequently, the approximate solution is obtained as follows:

$$
\begin{aligned}
u(x, t) & =\lim _{n \rightarrow \infty} u_{n}(x, t)=\lim _{n \rightarrow \infty} \sum_{k=0}^{n} \frac{e^{-x}(1+\mu)^{k}}{\Gamma(k \alpha+1)} t^{k \alpha} \\
& =e^{-x} E_{\alpha}\left((1+\mu) t^{\alpha}\right),
\end{aligned}
$$

where $E_{\alpha}\left((1+\mu) t^{\alpha}\right)$ is the Mittag-Leffler function.

Example 9. Now, the following time FADE subject to the initial condition is considered [21]:

$$
\begin{gathered}
\frac{\partial^{\alpha} u(x, t)}{\partial t^{\alpha}}=\kappa \frac{\partial^{2} u(x, t)}{\partial x^{2}}-v \frac{\partial u(x, t)}{\partial x}, \quad t>0,0<\alpha \leq 1, \\
u(x, 0)=\sin (x) .
\end{gathered}
$$


The corresponding iterative formula (20) for (25) can be derived as

$$
\begin{array}{r}
u_{n+1}(x, t)=u_{n}(x, t) \\
-\frac{1}{\Gamma(\alpha+1)} \int_{0}^{t}\left(\frac{\partial^{\alpha} u_{n}(x, \xi)}{\partial \xi^{\alpha}}+\nu \frac{\partial u_{n}(x, \xi)}{\partial x}\right. \\
\left.-\kappa \frac{\partial^{2} u_{n}(x, \xi)}{\partial x^{2}}\right)(d \xi)^{\alpha}
\end{array}
$$

Starting with $u_{0}(x, t)=u(x, 0)=x^{2}$, by formula (26), we derive the following results:

$$
\begin{aligned}
u_{1}(x, t)= & \sin (x)-(\nu \cos (x)+\kappa \sin (x)) \frac{t^{\alpha}}{\Gamma(\alpha+1)}, \\
u_{2}(x, t)= & \sin (x)-(\nu \cos (x)+\kappa \sin (x)) \frac{t^{\alpha}}{\Gamma(\alpha+1)} \\
& +\left(2 \kappa \nu \cos (x)+\left(\kappa^{2}-v^{2}\right) \sin (x)\right) \frac{t^{2 \alpha}}{\Gamma(2 \alpha+1)}, \\
u_{3}(x, t)= & \sin (x)-(\nu \cos (x)+\kappa \sin (x)) \frac{t^{\alpha}}{\Gamma(\alpha+1)} \\
& +\left(2 \kappa \nu \cos (x)+\left(\kappa^{2}-v^{2}\right) \sin (x)\right) \frac{t^{2 \alpha}}{\Gamma(2 \alpha+1)} \\
& +\left(\left(\nu^{3}-3 \kappa^{2} \nu\right) \cos (x)\right. \\
& \left.+\left(3 \nu^{2} \kappa-\kappa^{3}\right) \sin (x)\right) \frac{t^{3 \alpha}}{\Gamma(3 \alpha+1)}
\end{aligned}
$$

which is the same solution as that given in [21] using the ADM.

Example 10. Finally, the following nonhomogeneous time FADE with variable coefficients with the initial condition is considered:

$$
\begin{gathered}
\frac{\partial^{\alpha} u(x, t)}{\partial t^{\alpha}}=-x t \frac{\partial^{2} u(x, t)}{\partial x^{2}}+t \frac{\partial u(x, t)}{\partial x}+8 t x^{2}, \\
t>0,0<\alpha \leq 1, \\
u(x, 0)=x^{2} .
\end{gathered}
$$

Using (20), the iteration formula for (28) is given by

$$
\begin{array}{r}
u_{n+1}(x, t)=u_{n}(x, t) \\
-\frac{1}{\Gamma(\alpha+1)} \int_{0}^{t}\left(\frac{\partial^{\alpha} u_{n}(x, \xi)}{\partial \xi^{\alpha}}+x \xi \frac{\partial u_{n}(x, \xi)}{\partial x}\right. \\
\left.-\xi \frac{\partial^{2} u_{n}(x, \xi)}{\partial x^{2}}-2 \xi x^{2}\right)(d \xi)^{\alpha}
\end{array}
$$

Starting with $u_{0}(x, t)=u(x, 0)=x^{2}$, and using (29), we have

$$
\begin{gathered}
u_{1}(x, t)=x^{2}+\frac{8 x^{2} t^{\alpha+1}}{\Gamma(\alpha+2)}, \\
u_{2}(x, t)=u_{3}(x, t)=\cdots=u_{n}(x, t)=u_{1}(x, t) .
\end{gathered}
$$

Consequently, the exact solution is obtained as follows:

$$
u(x, t)=\lim _{n \rightarrow \infty} u_{n}(x, t)=x^{2}+\frac{8 x^{2} t^{\alpha+1}}{\Gamma(\alpha+2)} .
$$

\section{Conclusion}

The fundamental aim of this study was to obtain an analytical approximate solution of time FADEs using the FVIM. The aforementioned implementation indicates that this method is powerful and efficient in solving the equation in an easier and a more accurate way. The method also provides an analytical approximation solution in a rapidly convergent series with easily calculable terms for various physical problems. Therefore, FVIM is a more effective, a more convenient, and a more accurate method than other methods mentioned in the introduction. The obtained results denote that this method can be considered as an alternative to the other methods in the literature in terms of the purpose of solving linear or nonlinear FDEs in general.

\section{Conflict of Interests}

The authors declare that there is no conflict of interests regarding the publication of this paper.

\section{References}

[1] K. S. Miller and B. Ross, An Introduction to the Fractional Calculus and Fractional Differential Equations, Wiley, New York, NY, USA, 1993.

[2] S. G. Samko, A. A. Kilbas, and O. I. Marichev, Fractional Integrals and Derivatives: Theory and Applications, Gordon and Breach,, Yverdon, Switzerland, 1993.

[3] I. Podlubny, Fractional Differential Equations, Academic Press, New York, NY, USA, 1999.

[4] E. Çelik and M. Bayram, "The numerical solution of physical problems modeled as a systems of differential-algebraic equations (DAEs)," Journal of the Franklin Institute, vol. 342, no. 1, pp. 1-6, 2005.

[5] A. A. Kilbas, H. M. Srivastava, and J. J. Trujillo, Theory and Applications of Fractional Differential Equations, Elsevier, Amsterdam, The Netherlands, 2006.

[6] N. Guzel and M. Bayram, "Numerical solution of differentialalgebraic equations with index-2," Applied Mathematics and Computation, vol. 174, no. 2, pp. 1279-1289, 2006.

[7] J. Sabatier, O. P. Agrawal, and J. A. Tenreiro Machado, Eds., Advances in Fractional Calculus: Theoretical Developments and Applications in Physics and Engineering, Springer, Dordrecht, The Netherlands, 2007.

[8] M. Kurulay and M. Bayram, "Approximate analytical solution for the fractional modified $\mathrm{KdV}$ by differential transform method," Communications in Nonlinear Science and Numerical Simulation, vol. 15, no. 7, pp. 1777-1782, 2010. 
[9] M. Kurulay, B. A. Ibrahimoǧlu, and M. Bayram, "Solving a system of nonlinear fractional partial differential equations using three dimensional differential transform method," International Journal of Physical Sciences, vol. 5, no. 6, pp. 906-912, 2010.

[10] G. H. Zheng and T. Wei, "Spectral regularization method for a Cauchy problem of the time fractional advection-dispersion equation," Journal of Computational and Applied Mathematics, vol. 233, no. 10, pp. 2631-2640, 2010.

[11] M. M. Meerschaert and C. Tadjeran, "Finite difference approximations for fractional advection-dispersion flow equations," Journal of Computational and Applied Mathematics, vol. 172, no. 1, pp. 65-77, 2004.

[12] Z.-Q. Deng, V. P. Singh, and L. Bengtsson, "Numerical solution of fractional advection-dispersion equation," Journal of Hydraulic Engineering, vol. 130, no. 5, pp. 422-431, 2004.

[13] R. Metzler and J. Klafter, "Accelerating Brownian motion: a fractional dynamics approach to fast diffusion," Europhysics Letters, vol. 51, no. 5, pp. 492-498, 2000.

[14] M. E. Khalifa, "Some analytical solutions for the advection-dispersion equation," Applied Mathematics and Computation, vol. 139, no. 2-3, pp. 299-310, 2003.

[15] N. Pochai, "A numerical computation of a non-dimensional form of stream water quality model with hydrodynamic advection-dispersion-reaction equations," Nonlinear Analysis: Hybrid Systems, vol. 3, no. 4, pp. 666-673, 2009.

[16] A. R. Johnson, C. A. Hatfield, and B. T. Milne, "Simulated diffusion dynamics in river networks," Ecological Modelling, vol. 83, no. 3, pp. 311-325, 1995.

[17] D. A. Benson, S. W. Wheatcraft, and M. M. Meerschaert, "Application of a fractional advection-dispersion equation," Water Resources Research, vol. 36, no. 6, pp. 1403-1412, 2000.

[18] G. J. Fix and J. P. Roof, "Least squares finite-element solution of a fractional order two-point boundary value problem," Computers and Mathematics with Applications, vol. 48, no. 7-8, pp. 1017-1033, 2004.

[19] F. Liu, V. V. Anh, I. Turner, and P. Zhuang, "Time fractional advection-dispersion equation," Journal of Applied Mathematics and Computing, vol. 13, no. 1-2, pp. 233-245, 2003.

[20] Q. Huang, G. Huang, and H. Zhan, "A finite element solution for the fractional advection-dispersion equation," Advances in Water Resources, vol. 31, no. 12, pp. 1578-1589, 2008.

[21] S. Momani and Z. Odibat, "Numerical solutions of the spacetime fractional advection-dispersion equation," Numerical Methods for Partial Differential Equations, vol. 24, no. 6, pp. 1416-1429, 2008.

[22] F. Liu, P. Zhuang, V. Anh, I. Turner, and K. Burrage, "Stability and convergence of the difference methods for the space-time fractional advection-diffusion equation," Applied Mathematics and Computation, vol. 191, no. 1, pp. 12-20, 2007.

[23] R. K. Pandey, O. P. Singh, and V. K. Baranwal, "An analytic algorithm for the space-time fractional advection-dispersion equation," Computer Physics Communications, vol. 182, no. 5, pp. 1134-1144, 2011.

[24] R. K. Pandey, O. P. Singh, V. K. Baranwal, and M. P. Tripathi, "An analytic solution for the space-time fractional advectiondispersion equation using the optimal homotopy asymptotic method," Computer Physics Communications, vol. 183, no. 10, pp. 2098-2106, 2012.

[25] A. Golbabai and K. Sayevand, "Analytical modelling of fractional advection-dispersion equation defined in a bounded space domain," Mathematical and Computer Modelling, vol. 53, no. 9-10, pp. 1708-1718, 2011.
[26] F. Huang and F. Liu, "The fundamental solution of the spacetime fractional advection-dispersion equation," Journal of Applied Mathematics and Computing, vol. 18, no. 1-2, pp. 339350, 2005.

[27] A. R. Carella and C. A. Dorao, "Least-squares spectral method for the solution of a fractional advection-dispersion equation," Journal of Computational Physics, vol. 232, no. 1, pp. 33-45, 2013.

[28] G. Jumarie, "Table of some basic fractional calculus formulae derived from a modified Riemann-Liouville derivative for nondifferentiable functions," Applied Mathematics Letters, vol. 22, no. 3, pp. 378-385, 2009.

[29] G. Jumarie, "Modified Riemann-Liouville derivative and fractional Taylor series of nondifferentiable functions further results," Computers and Mathematics with Applications, vol. 51, no. 9-10, pp. 1367-1376, 2006.

[30] J.-H. He, "Approximate analytical solution for seepage flow with fractional derivatives in porous media," Computer Methods in Applied Mechanics and Engineering, vol. 167, no. 1-2, pp. 57-68, 1998. 


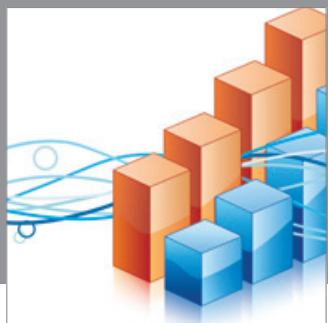

Advances in

Operations Research

mansans

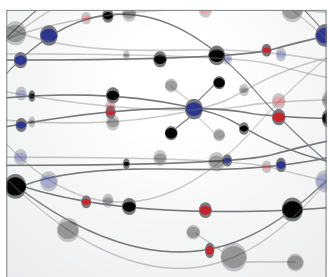

The Scientific World Journal
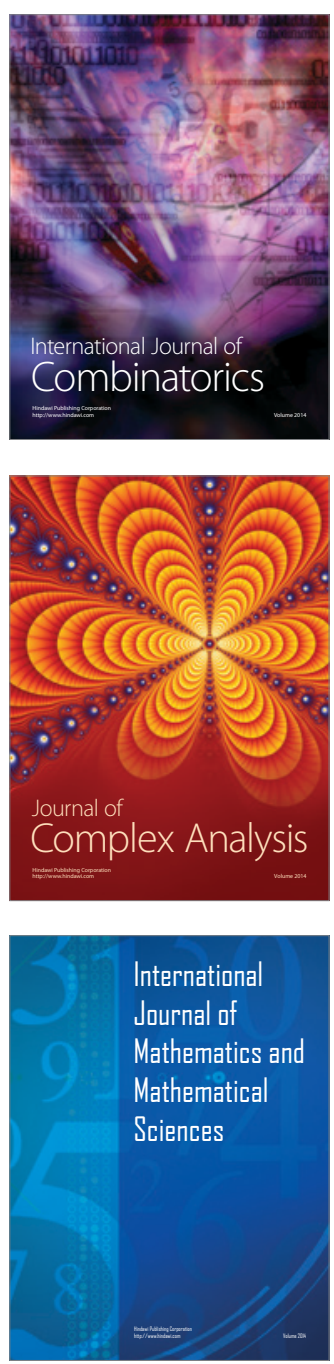
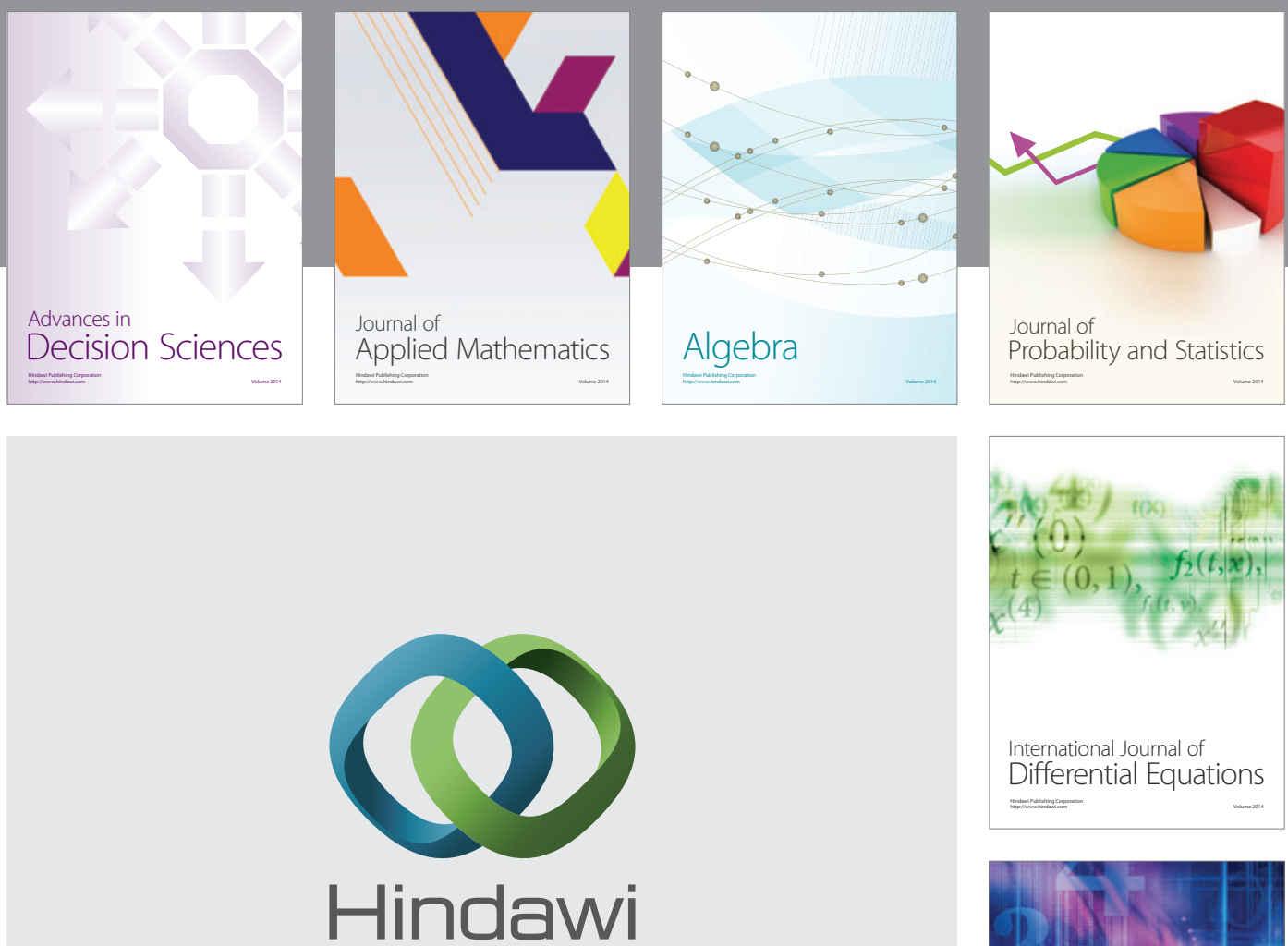

Submit your manuscripts at http://www.hindawi.com
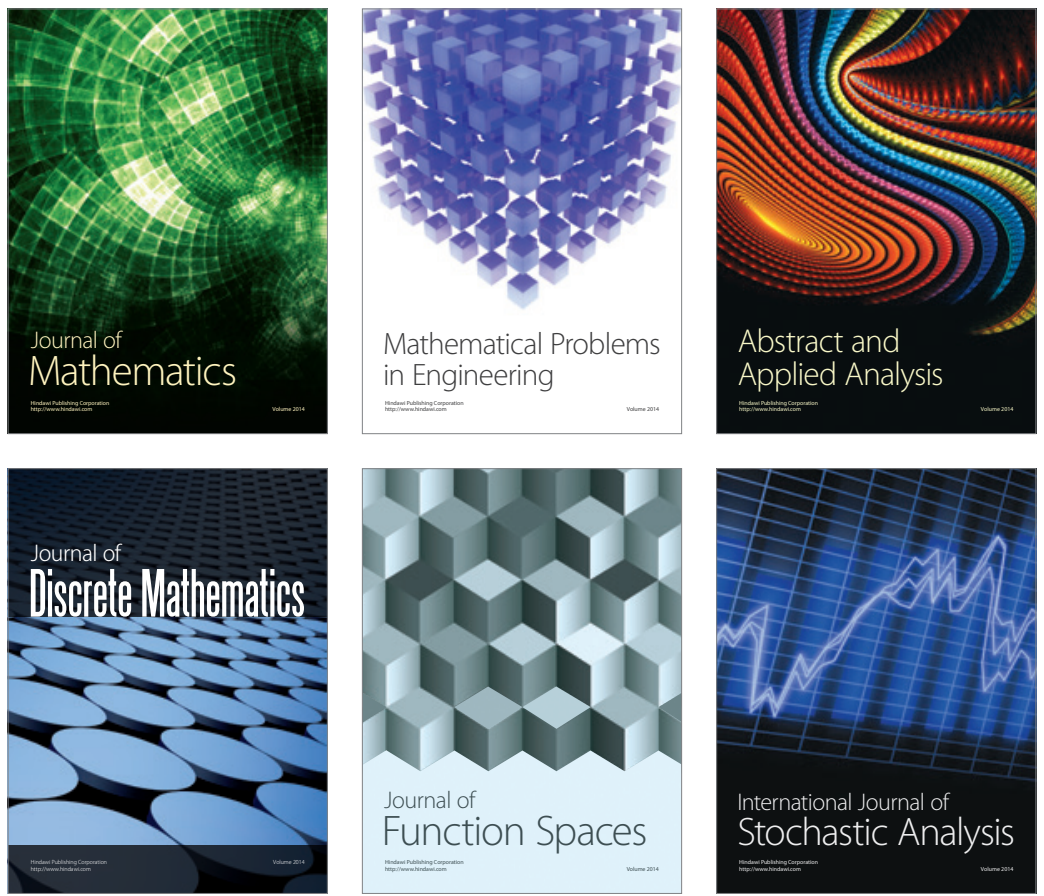

Journal of

Function Spaces

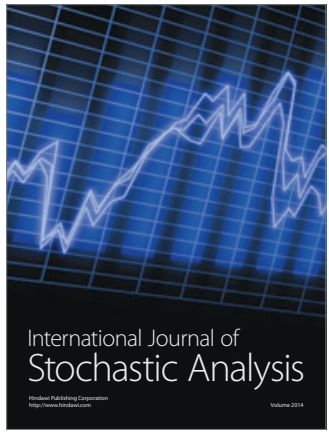

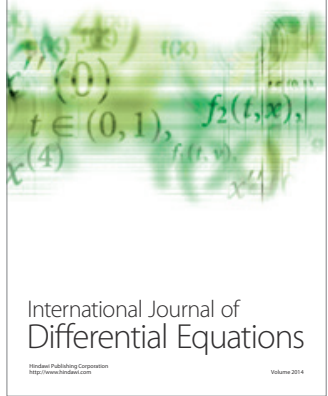
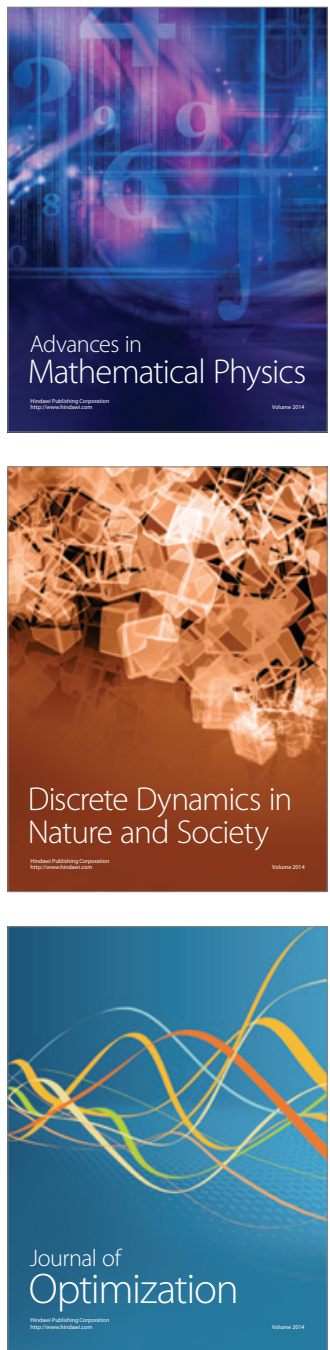